\title{
The Key Information Technology of Soybean Disease Diagnosis
}

\author{
Baoshi Jin ${ }^{1,2}$, Xiaodan $\mathrm{Ma}^{3}$, Zhongwen Huang ${ }^{4}$, and Yuhu Zuo ${ }^{5, *}$ \\ ${ }^{1}$ College of Agronomy Heilongjiang Bayi Agricultural University \\ DaQing China 163319 \\ ${ }^{2}$ General bureau of state farms of Heilongjiang Province Harbin China 150036 \\ ${ }^{3}$ College of Information Technology Heilongjiang Bayi Agricultural University \\ DaQing China 163319 \\ ${ }^{4}$ Academy of Sciences of Heilongjiang Land Reclamation Jia Musi China 154002 \\ ${ }^{5}$ College of Agronomy Heilongjiang Bayi Agricultural University \\ DaQing China 163319 \\ zuoyhu@163.com
}

\begin{abstract}
Combining image processing and artificial neural network technology, a new diagnose method of soybean leaf diseases has been proposed, which identified the methods of division algorithm and eigenvalue computation, meanwhile, established a three-level neural network model to identify the diseased spot areas. According to the characteristics of soybean leaf disease, a new diagnose method has been formed, through extraction of geometric features, color and texture feature, which provided reference to Remote Intelligent diagnosis of soybean leaf disease.
\end{abstract}

Keywords: Soybean, Leaf Diseases, Image Processing, Neural network, Diagnose.

\section{Introduction}

At present, The image processing technology has become an important agricultural research content of digital agricultural information detection, the disease type, extent and damage control methods can be passed to agricultural producers synchronously after the disease images have been processed, segmented and recognized using image processing technology, through which, the diseases can be diagnosed automatically, comprising to traditional diagnose method, the method based on image processing technology has many advantages such as simple, rapid, real-time, objective, accuracy etc.

For the most of plants, the diseases can be reflected through its lamina at a great degree, so it is possible to get affected condition through researching laminas, and the identification of disease spots and its associated feature information become the basis

* Corresponding author.

The project supported by General bureau of state farms of Heilongjiang Province (HNK11A-06-02-02). 
for judging Diseases. As early as 1995, Tao[1] studied on color differences between apples and potatoes using machine vision, Zhou-Long[2] detected the image edge and Shen Zuo-rui[3] distinguished the geometrical characteristic among images using fuzzy enhancement and mathematics morphology separately.

Since the mid-20th century, 80, the image processing technology has been widely used in agricultural engineering studies. after retrieving a large number of crop diseases diagnosis technology[4-12], we found that, Ma Xiaodan[13] etc has achieved automatic recognition of soybean leaf diseased spots using neural network technology, CaoMin[14] has achieved determination of soybean nitrogen using image processing technology, Mari Oide[15] has recogonized 364 leaf shapes of 38 soybean varieties using Hopfield pattern recognition which did not require any shape feature extraction, the neural network determined right and wrong. in summary, soybean leaf disease diagnosis technology based on image processing and neural network have not been reported yet.

This article will focus on technology based on image processing and neural network to diagnose the soybean leaf diseases, in the field environment, through the machine vision to obtain image of leaf disease samples, to achieve automatic identification of disease, computer image processing and pattern recognition technology are proposed to diagnose the soybean leaf diseases, furthermore, has become the basement for remote intelligent diagnosis of soybean diseases.

\section{Materials and Methods}

\subsection{Image Acquisition}

The samples of soybean leaf diseases have been collected in open environment of field, which used non-destructive collection method, in natural light, soybean leaves have been loaded in home-made loading template, and used DSLR- $\alpha 350$ SONY digital cameras wih 14.2 million effective pixels to collect the images which would be stored into soybean leaf disease image database with JPEG format.

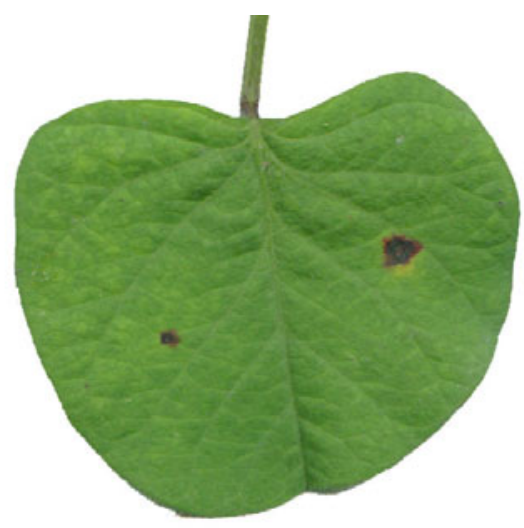

Fig. 1. The image of diseased leaf 


\subsection{Pre-processing of Plant Disease Image}

Image of plant disease leaf could be affected by various noise sources in the generation and transmission which would lead to quality degradation. It is impossible to describe statistical model using random process, thus, this article used a local smoothing method to eliminate image noises, which named median filter approach.

A median filter window may be square $(m \times m)$, rectangular $(m \times n)$ or cross-shaped etc.

Firstly, Median filter will sort the gray value of template pixel, while $\mathrm{n}$ is odd number, the gray value of pixel Located in the middle is called the median number of these $n$ numbers., while $\mathrm{n}$ is even number, the gray value of pixel Located in the middle of the average of the two values is called the median number, the expression is as follows:

$$
g(i, j)=\operatorname{median}\left[f_{A}(i, j)\right]
$$

Where, the median represents the middle value of the window, $f_{A}(i, j)$ represents the gray value of pixel.

\subsection{Recognition of Diseased Spots}

BP network is one of the most widely used class of models, among of which the error is back propagation. From the topology point of view, BP network is a typical hierarchical network structure, is divided into input layer, hidden layer and output layer, between layers generally use the whole interconnect, the figure 1 is a three-layer BP neural network, i, j, k are input node, output node and hidden node separately.

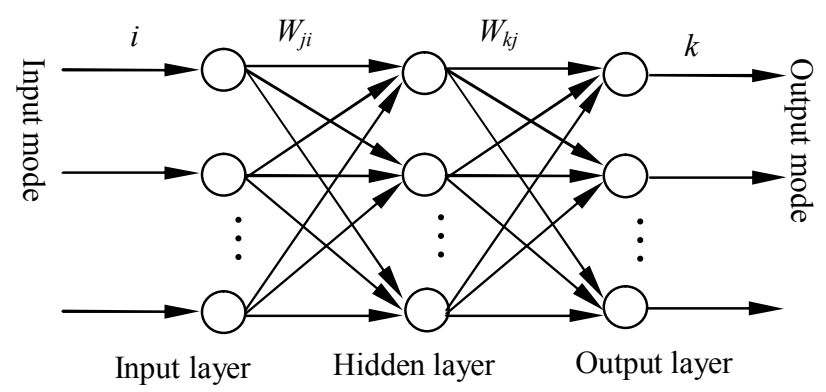

Fig. 2. Three-Layer BP network structure diagram

The input layer receives external input mode, and has been transmitted by the input node to the nodes of hidden layer. The hidden layer is a layer of internal processing units of BP network and pattern recognition has mainly achieved by the hidden layer nodes. Assume that there is a input mode $X_{p}=\left\{X_{p i}\right\}$, the each output of hidden layer nodes can be expressed as: 


$$
I_{p j}=\sum_{i} w_{j i} O_{p i}
$$

Where, $w_{j i}$ is the connection weights between input layer and hidden layer nodes, $O_{p i}$ is the output value of input node, the output of hidden layer is expressed as follows:

$$
O_{p j}=\frac{1}{1+e^{-I_{p j}}}
$$

Output layer produces the final classification result, that is the output mode, the output node of input layer is as follows:

$$
I_{p k}=\sum_{j} w_{k j} O_{p j}
$$

$w_{k j}$ is connection weights between the node $j$ of hidden layer and node $k$ of output layer, $O_{p j}$ is the output value of node $j$ of hidden layer. The output of node $k$ is as follows:

$$
O_{p k}=\frac{1}{1+e^{-I_{p k}}}
$$

For the attraction of disease pathogen, the color of diseased spots area has often been influenced in a certain degree, which different with healthy area of lamina obviously, thus, the color characteristics of diseased area and healthy area would be used as input values, concretely involves $R, G, B$ values of each pixel; In the course of the study, choosing ten pixels as training samples, of which five samples of diseased spots used as input value need to be extracted and another five samples of healthy area as input values should be wiped out, and then, the effective network weights could be got though a series complicated evolution process including copy, cross and variation over and over again, and saved to weight file at last.

The values of $R, G$ and $B$ component in each pixel were listed out in table 1 , of which the color values of sample points one to five represented that of diseased spots, and anther five sample points six to ten represented that of background area.

Fig. 3. Soybean leaf diseased spot Image before and after recognized 


\subsection{Feature Calculation}

\section{1) Geometric Feature[16]}

The characteristic parameters of the lesion is very important to determine the diseases, This region labeling and contour tracking has been used to calculate the geometric characteristics of lesion area.

Table 1. Values of Geometric Feature

\begin{tabular}{ccccccc}
\hline No. & S & L & $c$ & $e$ & $E$ & $s$ \\
\hline 1 & 278 & 62 & 0.909 & 13.827 & 0.844 & 0.576 \\
2 & 1311 & 139 & 0.853 & 14.738 & 0.950 & 0.638 \\
$\ldots \ldots$ & $\ldots \cdots$ & $\cdots \cdots$ & $\ldots \cdots$ & $\ldots \cdots$ & $\ldots \cdots$ & $\cdots \cdots$ \\
\hline
\end{tabular}

\section{2) Color Feature [17]}

While diagnosis of crop diseases, the HSI model has great advantages, HSI model can reduce the light intensity to determine the impact of color, therefore, this study extract the color feature in HSI color space, therefore, RGB space need to converted to HSI color space.

Table 2. Values of Color Feature

\begin{tabular}{cccc}
\hline No. & $H$ & $S$ & $I$ \\
\hline 1 & 64.71 & 32.53 & 78.21 \\
2 & 69.72 & 33.00 & 66.52 \\
$\ldots \ldots$ & $\ldots \ldots$ & $\ldots \ldots$. & $\cdots \cdots$ \\
& & & \\
\hline
\end{tabular}

\section{3) Texture Feature [18]}

The image of disease leaf includes not only geometric information, color information, but also contains the texture information, fiom which many valuable information can be abtained. Because different types of disease pathogens can produce different diseases, the lesion area showes different texture, thus, the texture features can provide an important basis for identification of disease. 
Table 3. Values of Texture Feature

\begin{tabular}{ccccc}
\hline No. & $m$ & $R$ & $\mu_{3}$ & $U$ \\
\hline 1 & 245.52 & 0.02 & -3.61 & 0.70 \\
2 & 226.81 & 0.06 & -8.00 & 0.47 \\
$\ldots \ldots$ & $\ldots \ldots$ & $\ldots \ldots$ & $\ldots \ldots$ & $\ldots \ldots$ \\
\hline
\end{tabular}

\section{Result and Discussion}

From figure 1, we see that the color of soybean brown spot is obviously different to that of healthy area, so it is feasible to use color characteristic as input value of the network, and the most of the diseased spots shown in figure 4 have been extracted, especially the bigger ones with distinct characteristic, so the algorithm accuracy can reach to more than $90 \%$, furthermore, the main influenced factor lay in some aspects bellow:

1) The first factor is the number of samples, how many samples should be chosen depends on the complexity of the lamina itself, that is to say, the more complex types of diseased lamina, the more samples should be chosen.

2) The second factor is the resolution of the digital camera, which influenced the extraction accuracy of diseased spots.

3) The third factor is the light intensity which led to the loss of the image color, so reducing the interference of human factor and environmental factors should be taken into account when collecting the lamina images.

\section{Conclusion}

Based on the colorimetric mechanism, the soybean brown spots have been recognized successfully through training the artificial neural network, the method proposed in this paper overcomes the shortcomings of traditional threshold method. The experiments result shows that the precondition for accuracy reached to $100 \%$ is choosing enough samples seriously and combining with image processing technology.

In the paper, we have studied the key technology of Intelligent diagnosis of crop diseases. Firstly, proposed the image acquisition method, secondly, determined the preprocessing algorithms of diseased leaves image and the segmentation algorithm of soybean leaf lesion, at last, pre-research the Identification model of soybean leaf disease from the geometrical features, color and texture characteristic respectively, which provide the basement for intelligent diagnosis of diseased soybean leaf.

This study which can be applied not only in recognizing of soybean diseased spots, but also in that of other farm crop, such as rice, maize have been popularized in practical life and production, meanwhile, provides a practical scientific method for agriculture researchers and solid technology basement for species identification, characteristic recognition and diseased diagnosis. 


\section{References}

[1] Tao, Y.: Machine vision for color inspection of potatoes and apples. Transactions of the ASAE 38, 555-1561 (1995)

[2] Zhou, L.: Research on Fuzzy Detection Method of Pest's Image in Stored Grain Based on Machine Vision. Computer Applications and Software (8), 24-25 (2005)

[3] Shen, Z.-R.: Use of math-morphological features in insect taxonomy

[4] Dong, J.: Agricultural Plant Pathology. China Agricultural Press (2001) (in Chinese)

[5] Lai, J., Li, S., Ming, B., Wang, N., Wang, K., Xie, R., Gao, S.: Advances in Research on Computer-Vision Diagnosis of Crop Diseases. Scientia Agricultura Sinica 42(4), 1215-1221 (2009)

[6] Georgieva, K., Georgieva, Y., Daskalov, D.: Theoretical substantiation of model of system for evaluation a state of vine plants and taking a decision for plant protection activities. Trakia Journal of Sciences (Series Social Sciences) 1, 30-32 (2003)

[7] Ying, Y., Zhang, W., Jiang, Y.: Machine vision technology in the agricultural harvest and process automation application. Agricultural Machinery 31(3), 112-115 (2000) (in Chinese)

[8] Shanwen, H.: Image processing technology in environmental applications. Electric Association Journal Featured, 455-458 (1985)

[9] Panigrahi, S.: Background Segmentation and Dimensional measurement of Corn Germplasm. Transactions of the ASAE 38(1), 291-297 (1995)

[10] Sasaki, Y., Okamoto, T., Imou, K., Tor, T.: Automatic Diagnosis of Plant Disease. Journal of JSAM 61(2), 119-126 (1999)

[11] Sasaki, Y., Suzuki, M.: Construction of the Automatic Diagnosis System of Plant Disease Using Genetic Programming Which Paid Its Attention to Variety. ASAE Meeting Presentation, paper No. 031049 (2003)

[12] Sanyala, P., Patel, S.C.: Pattern recognition method to detect two diseases in rice plants. The Imaging Science Journal 56(6), 319-325 (2008)

[13] Ma, X., Qi, G.: Investigation and recognition on diseased spots of soybean leaf based on neural network. Journal of Heilongjiang First Land Reclamation University 18(2), 84-87 (2006) (in Chinese)

[14] Cao, M.: Detecting Research of Soybean's Plant Nitrogen Based on image processing Technology. Jilin University, Changchun (2005) (in Chinese)

[15] Oide, M., Ninomiya, S.: Discrimination of soybean leaflet shape by neural networks with image input. Computers and Electronics in Agriculture, 59-72 (October 2000)

[16] Liu, S.: Based on fuzzy neural network diagnostic system of grape disease. Agricultural Engineering 9, 144-147 (2006) (in Chinese)

[17] Liu, S.: Based on fuzzy neural network diagnostic system of grape disease. Agricultural Engineering 9, 144-147 (2006) (in Chinese)

[18] Zhao, F., Zhao, R.: Texture segmentation and feature extraction Methods. Chinese Journal of Stereology and Image Analysis 3(4), 238-245 (1988) (in Chinese) 